\title{
Plant expression systems for production of hemagglutinin as a vaccine against influenza virus
}

\author{
Patrycja Redkiewicz, Agnieszka Sirko, Katarzyna Anna Kamel\# and Anna Góra-Sochacka \\ Institute of Biochemistry and Biophysics, Polish Academy of Sciences, Warsaw, Poland
}

\begin{abstract}
Many examples of a successful application of plantbased expression systems for production of biologically active recombinant proteins exist in the literature. These systems can function as inexpensive platforms for the large scale production of recombinant pharmaceuticals or subunit vaccines. Hemagglutinin (HA) is a major surface antigen of the influenza virus, thus it is in the centre of interests of various subunit vaccine engineering programs. Large scale production of recombinant $\mathrm{HA}$ in traditional expression systems, such as mammalian or insect cells, besides other limitations, is expensive and time-consuming. These difficulties stimulate an ever-increasing interest in plant-based production of this recombinant protein. Over the last few years many successful cases of HA production in plants, using both transient and stable expression systems have been reported. Various forms of recombinant HA, including monomers, trimers, virus like particles (VLPs) or chimeric proteins containing its fusion with other polypeptides were obtained and shown to maintain a proper antigenicity. Immunizations of animals (mice, ferrets, rabbits or chickens) with some of these plant-derived hemagglutinin variants were performed, and their effectiveness in induction of immunological response and protection against lethal challenge with influenza virus demonstrated. Plant-produced recombinant subunit vaccines and plant-made VLPs were successfully tested in clinical trials (Phase I and II) that confirmed their tolerance and immunogenicity.
\end{abstract}

Key words: recombinant hemagglutinin, avian influenza, plant-produced, VLP, ELISA

Received: 10 June, 2014; revised: 15 July, 2014; accepted: 01 September, 2014; available on-line: 09 September, 2014

\section{INTRODUCTION}

Currently licensed influenza vaccines are produced in embryonated chicken eggs. For many decades these vaccines have been successfully used and proven to be safe and effective. Their production system has some limitations, such as a need for specific pathogen-free (spf) embryonated eggs, time needed for adaptation to the new circulating virus subtypes, long production time, and limited capacity (Cummings et al., 2014). These limitations became evident when swine H1N1 influenza pandemic emerged unexpectedly, while the main attention was focused on the H5N1 virus. It is now obvious that there is a great demand for development of new technologies to engineer recombinant vaccines with capacity for easy scaling up and rapid production. Taking into account the high mortality rate of poultry caused by highly pathogenic avian influenza virus $\mathrm{H} 5 \mathrm{~N} 1$, the necessity of flock elimination and resulting economic losses, effective and inexpensive vaccines intended not only for humans but also animals are needed. Many countries develop alternative platforms for production of large amounts of safe influenza vaccines based on mammalian (Vero, MDCK), chicken (CEF), insect (Sf9) or bacterial (Escherichia coli) cells (Kanagarajan et al., 2012; www.who.int). However, production of subunit vaccines on a large scale using these systems is still expensive and unprofitable.

Plant-based expression systems offer several advantages, such as time and cost efficiency, lower risk of contamination from human pathogen and greater scalability in comparison to the conventional systems (Obembe et al., 2011). They can be divided into two main types, stable and transient (Fig. 1). In stable transformants, transgenes are incorporated into either the nuclear genome (transgenic plants) or the chloroplast genome (transplastomic plants) (Obembe et al., 2011). A major advantage of stably transformed plants is that the heterologous protein production is heritable, which allows simple and rapid scaling-up. However, relatively long time (1-2 years) is required to obtain stabilized transformants, and often the production level of recombinant proteins might be lower than expected. The most popular method of creating stable transformants is based on Agrobacterium-mediated transformation.

Transient expression systems may be used not only for quick validation of the plant expression cassettes but also for production of considerable amounts of recombinant proteins within a short time. Transient expression systems can be based on: (i) infiltration of plants with Agrobacterium carrying a binary vector, (ii) infection of plants with a modified plant virus or (iii) combination of both, the viral vector-based expression and Agrobacteriummediated method (e.g. MagnICON ${ }^{\circledR}$ and 'Launch vector' system). All variants of transient expression systems are much faster than the stable transformation.

e-mail: annag@ibb.waw.pl

\#Present address: Department of Genomics, Institute of Plant Genetics, Polish Academy of Sciences, Poznań, Poland

Abbreviations: CaMV, Cauliflower mosaic virus; CEF, chicken embryonic fibroblasts; CPMV, Cowpea mosaic virus; ELISA, enzyme linked immunosorbent assay; ELP, elastin-like polypeptide; ER, endoplasmatic reticulum; Fc, crystallizable fragment of an antibody; FW, fresh weight; $\mathrm{HA}$, hemagglutinin; $\mathrm{HI}$, hemagglutination inhibition; IFNy, interferon gamma; IgG, immoglobulin G; KDEL, ER retention signal; MDCK, Madin-Darby canine kidney cells; MN, microneutralisation; M1, matrix protein; NA, neuraminidase; Sf9Spodoptera frugiperda Sf21 ovary cells; spf, specific pathogen free; SHR, single radial hemolysis; UTR, untranslated region; VLP, virus like particles; VN, virus neutralization. 


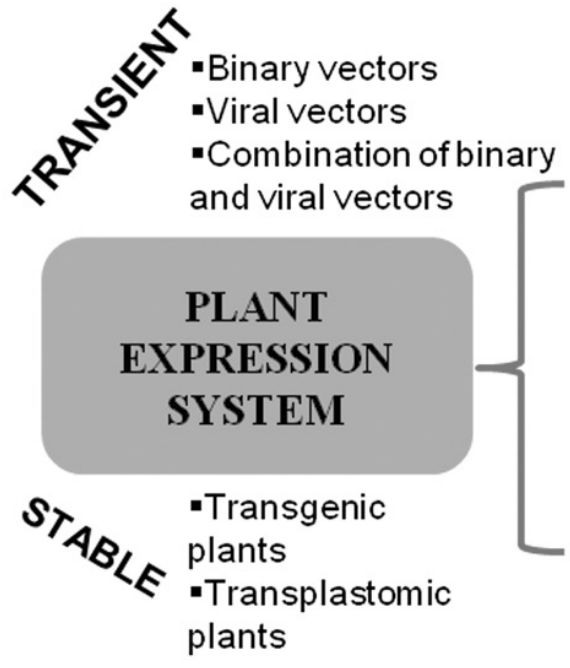

transformation time: a few weeks

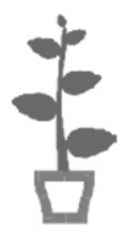

infiltration

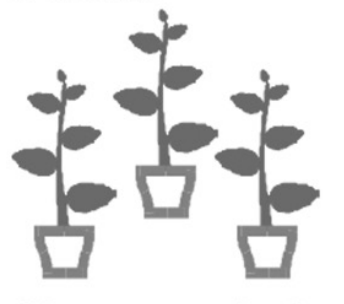

biomass production

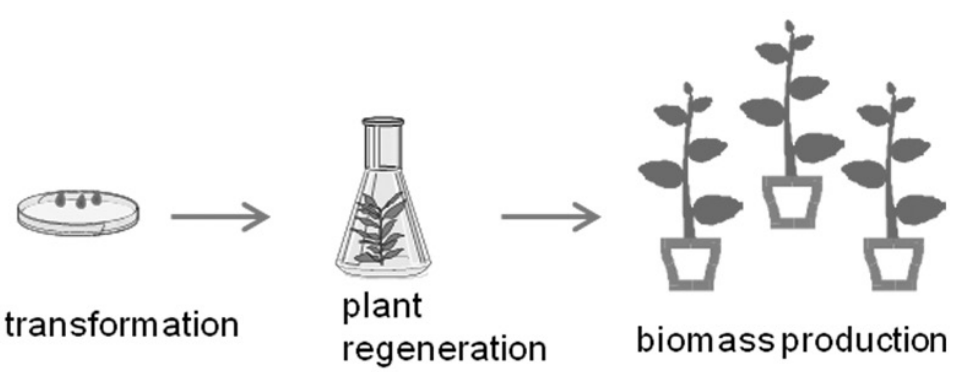

time: 1-2 years

Figure 1. Comparison of two main types of plant expression systems.

The growing interest in vaccines against influenza virus resulted in intensification of work focusing on production of the virus antigens in plant-based systems. Two surface proteins of influenza viruses, hemagglutinin (HA) and neuraminidase (NA), are the primary antigens that elicit immunological response after infection. HA is considered to be a stronger antigen than NA, thus it is the main object of interest for researchers working on recombinant vaccines against influenza. HA is synthesized as a precursor polypeptide HAO in the endoplasmic reticulum, where it is assembled into trimers. Then, via the Golgi network it is exported to the cell surface where it is cleaved by host proteases into two subunits: HA1 and HA2. The HA1 subunit forms a globular head which contains the receptor binding site, a main target for neutralizing antibodies (Stevens et al., 2006).

The overall scheme of experiments related to the production of subunit vaccines against influenza in plant-based systems is explained in Fig. 2. Various experimental strategies were used to improve the expression of HA in plants and to enhance the immunogenicity of the plant-produced variants of HA. As it is schematically shown in Fig. 3, these strategies can be divided into those dependent and independent on the plant expression system. The first group includes modifications of elements of the expression cassettes (mainly in plasmid vectors), improvement of the yield of amplification and facilitation of handling (mainly in virus-based vectors). The second group includes modifications of the target sequences leading to increased translation (due to codon optimization) or increased protein accumulation (due to different truncated or elongated variants or due to a different subcellular location).

Below, we shortly discuss published reports on successful HA production in plant systems listed in Table 1. We describe several most promising transient and stable systems used for this purpose, next we refer the strategies used for antigenic protein purification and finally, we focus on the clinical trials of plant-based vaccine formulations.

\section{TRANSIENT EXPRESSION}

Several kinds of transient expression systems were used for efficient production of influenza virus antigens. In all of them Agrobacterium was either infiltrated using a syringe (without a needle), into the underside of leaves or vacuum infiltrated using the whole plant. The second method, more efficient and convenient, is usually used for a large scale production. Nicotiana benthamiana plants are routinely used for transient expression.

\section{'Launch vector' system}

This advanced transient expression system combines the elements of plant RNA viral vectors (based on tobacco mosaic virus, TMV) and Agrobacterium binary plasmids. The system was developed by a group from Fraunhofer USA Center for Molecular Biotechnology. The hybrid launch vector, pBID4, contains the 35S promoter from a cauliflower mosaic virus (35S CaMV) that drives initial transcription of the recombinant viral genome, the nos terminator, genes for virus replication and cell-to-cell movement protein, and the target gene cloned under the transcriptional control of the coat protein subgenomic mRNA promoter. Following agroinfiltration, multiple copies of the viral vector sequences are produced (replicated) within the plant cells. The cloned target genes are then amplified along with the viral sequences and they are expressed from multiple copies (Musiychuk et al., 2007).

This system was used for production of HA ectodomain from different influenza virus strains: H3N2 (A/ Wyoming/03/03), H5N1 (A/Indonesia/05/05), H5N1 A/Bar-headed Goose/Qinghai/1A/05 and H5N1 A/ Anhui/1/05 (Shoji et al., 2009a; Shoji et al., 2009b; Shoji et al., 2008). The sequences were optimized for plant 


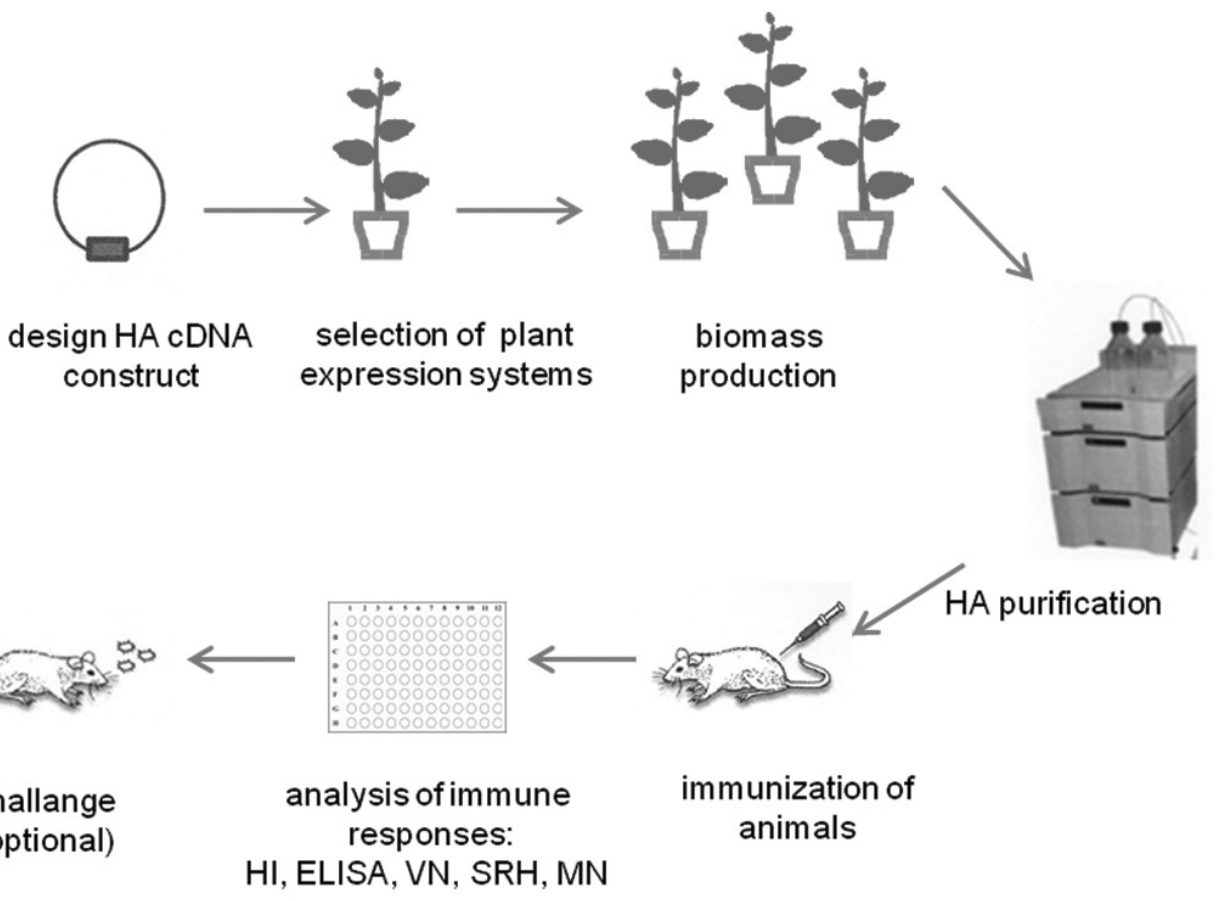

Figure 2. Scheme explaining production of recombinant vaccines in plants. $\mathrm{HI}$, hemagglutinin inhibition; ELISA, enzyme linked immunosorbent assay; VN, virus neutralization; SHR, single radial hemolysis; MN, microneutralisation.

expression and were encoding the truncated HA without the transmembrane domain but with endoplasmic reticulum retention signal (KDEL) and the polyhistidine affinity purification tag (6xHis) at the C-terminus. The native signal peptide was either removed or replaced by the PR-1a (tobacco pathogenesis-related protein) signal peptide. The yield of HA production was different depending on HA subtype, for example the highest level of HA H3 achieved on the seventh day post-infiltration was $200 \mathrm{mg} / \mathrm{kg}$ of tobacco leaves FW. Western blot analysis indicated that HA was produced as an intact (uncleaved) monomer. Following confirmation of its antigenicity by ELISA and a single-radial immunodiffusion (SRID) assay, immunization of Balb/c mice was performed. Recombinant antigens along with Quil A (saponin-based adjuvant) were injected subcutaneously in three doses, two weeks apart. The vaccine induced both, humoral (HA specific IgG) and cellular (IFN $\gamma$ and interleukin 5) immune responses (Shoji et al., 2008). The sera of mice immunized even with the lowest dose of recombinant HA H3 $(5 \mu \mathrm{g})$ gave positive results in hemagglutination inhibition (HI) and virus neutralization (VN) tests with the homologous H3N2 influenza virus (Shoji et al., 2008). Similarly, all Balb/c mice and $70 \%$ of ferrets immunized with $\mathrm{H} 5 \mathrm{HA}$ (A/Indonesia/05/05) elicited serum $\mathrm{HI}$ antibodies already after the second dose. Moreover, immunized ferrets were fully protected against challenge with the homologous virus (Shoji et al., 2009a). Cross-clade HI antibody reactivity was evaluated by analysis of sera collected from mice groups immunized with either HA H5 from clade 2.2 (A/Bar-headed Goose/ Qinghai/1A/05) or clade 2.3 (A/Anhui/1/05). Both recombinant HA variants elicited reciprocal cross-clade reactivity, while serum $\mathrm{HI}$ antibodies against clade 1 (A/ Vietnam/1194/04) and clade 2.1 (A/Indonesia/05/05) were not detected (Shoji et al., 2009b).

Similar experiments were done with HAs from the strains comprising the 2008-2009 seasonal vaccine
(A/Brisbane/10/07, A/Brisbane/59/07 and B/Flori$\mathrm{da} / 04 / 06$ ) and California/04/09 (H1N1) (Shoji et al., 2012). Depending on the HA variant, the level of recombinant proteins reached $400-1300 \mathrm{mg} / \mathrm{kg} \mathrm{FW}$, and the solubility was $>70 \%$. The recombinant HA (HAC1) from California/04/09 (H1N1) was used as a vaccine along with a silica nanoparticle-based (SiO2) drug delivery system and/or mucosal adjuvant candidate bis- $\left(3^{\prime}, 5^{\prime}\right)$ cyclic dimeric guanosine monophosphate (c-di-GMP). Mice were immunized by intratracheal route. Interestingly, the double-adjuvanted vaccine induced high systemic antibody responses, which were similar to the ones induced by the control systemic vaccination (intraperitoneally) with HAC1 adsorbed on aluminum hydroxide (Alum) (Neuhaus et al., 2014).

Recent improvement of the expression systems resulted in production of HA antigen in a trimeric form, which might mimic the native HA structure (Shoji et al., 2013). This should enhance immunogenicity and increase stability of the recombinant protein. Trimeric forms of HA (tHA-BC) from A/California/04/09 strain were generated by introducing the trimerization motif from an actin binding protein, coronin, at the C-terminus of HA. Immunization of mice with this antigen in the presence of adjuvant Alhydrogel ${ }^{\circledR}$ (aluminium hydroxide gel) induced specific anti-HA neutralizing antibodies and protected mice against a lethal dose of mouse-adapted A/California/04/09 (MA-CA/04/09) virus used in the challenge experiment. As expected, immunogenic and protective doses of the trimeric form (tHA-BC) were lower than those reported earlier for the monomeric HA (HAC1).

This production system was also used to generate fusions consisting of recombinant HA and a carrier molecule, an engineered enzyme lichenase (LicKM). The LicKM enzyme is derived from the thermostable enzyme, $\beta-1,3-1,4$-glucanase from Clostridium thermocellum and was used previously as a carrier molecule for report- 


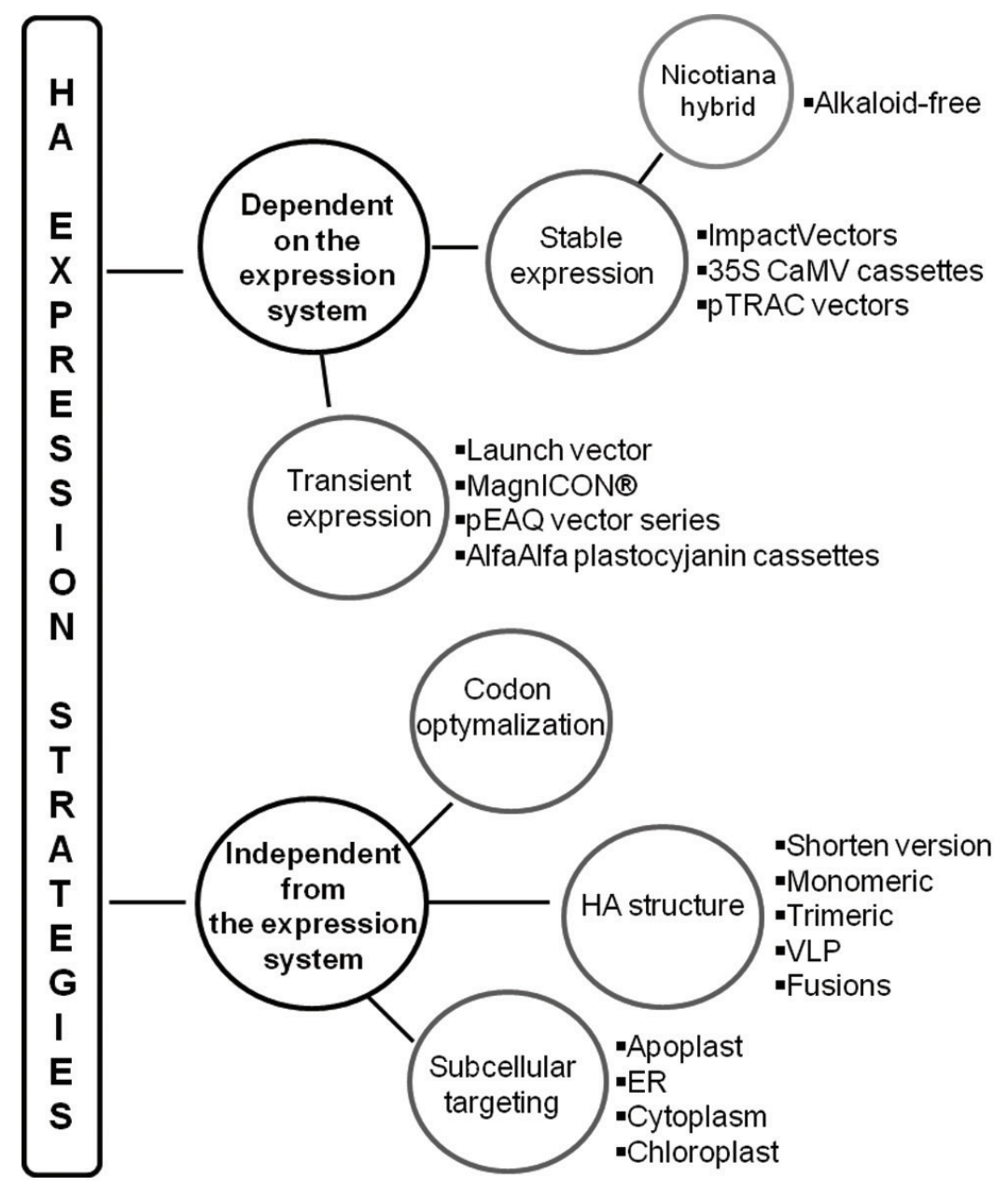

Figure 3. Plant-based HA expression strategies discussed in this work.

er gene expression in prokaryotic and eukaryotic systems (Goldenkova et al., 2002). The LicKM enhanced expression of recombinant proteins and could be used for easy and cost-effective recovery of the target protein. It was reported that 10 -min heat treatment at $65^{\circ} \mathrm{C}$ removes up to $50 \%$ of contaminating plant proteins while leaving LicKM fusions unaffected (Musiychuk et al., 2007). The launch vector/lichenase carrier system has been applied for efficient production of many recombinant variants of HA proteins. These fusion proteins include LicKMH5SD and LicKM-H5GD containing the stem domain, SD (17-58 aa followed by a triple glycine linker and 293-535 aa), and the globular domain, GD (59-292 aa) of HA from A/Vietnam/04 H5N1 virus, respectively, as internal fusion with LicKM.

The expressed HA fusions were recognized by serum raised against HA from the A/Vietnam/04 influenza virus (Musiychuk et al., 2007). Production and evaluation of HA domains (SD and GD) of influenza virus $\mathrm{A} / \mathrm{W}_{\mathrm{y}}$ oming/03/03 strain expressed as fusions to the LicKM was also reported (Mett et al., 2008). Additionally, one NA domain (38-468 aa) was expressed. In all constructs the signal peptide of PR1a and 6xHis plus KDEL motif were included at the $\mathrm{N}$-terminus and $\mathrm{C}$-terminus, respectively. Production level was about $100 \mathrm{mg} / \mathrm{kg} \mathrm{FW}$ in the case of LicKM-SD and LicKM-GD, whereas for NA it was higher $(300 \mathrm{mg} / \mathrm{kg} \mathrm{FW})$. The plant-produced HALicKM fusion was highly immunogenic and protected immunized ferrets against infection with a homologous virus. Enhancement of the immune response in ferrets was observed after addition of recombinant NA (Mett et al., 2008).

\section{MagnICON $^{\circledR}$ system}

Plant transient expression system - MagnICON ${ }^{\circledR}$ (ICON Genetics GmbH, Halle, Germany) is based on in planta assembly of functional viral vectors from two separate 5' and 3' pro-vector modules. Agrobacterium cells are used to deliver various modules that are subsequently assembled inside the plant cells with the help of a site-specific recombinase (Marillonnet et al., 2004, Gleba et al., 2005). Thus, the system combines advantages of Agrobacterium-mediated transfection (high efficiency) with the high speed and expression level of viral vectors.

This system was used to obtain HA-based vaccine for chickens against H5N1. The full-length H5 HA from the reassortant virus strain NIBRG-14 was targeted to different cell compartments like cytoplasm, chloroplast and apoplast (Kalthoff et al., 2010). The highest level of recombinant protein $(300 \mathrm{mg} / \mathrm{kg} \mathrm{FW})$ was achieved in the case of apoplast targeting, especially when the signal peptide of tobacco calreticulin was used. Recombinant HA was highly immunogenic and its application with adjuvant fully protected chickens against a lethal challenge infection with heterologous H5N1 (A/whooper swan/ Germany/R65/2006) virus.

The MagnICON ${ }^{\circledR}$ system has been used also by our group. The aim was to obtain an efficient production of the recombinant HA1 subunit of HA (17-338 aa) 

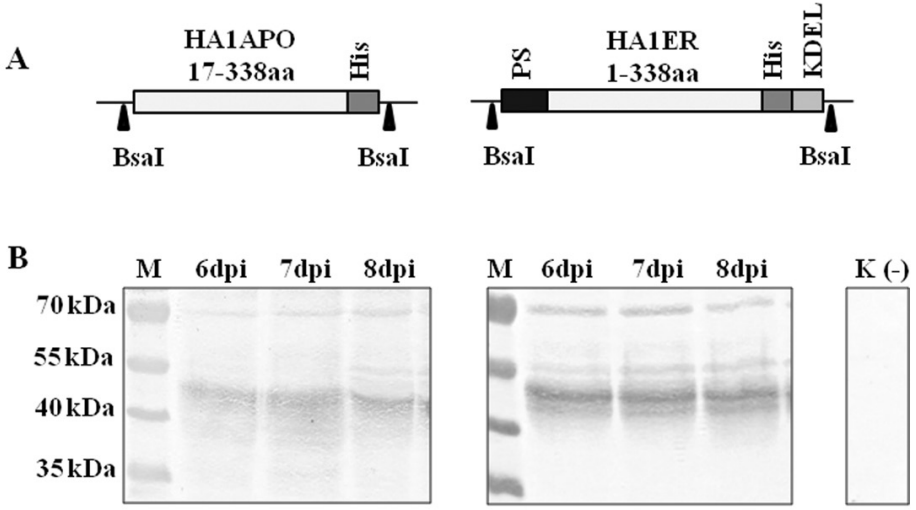

C

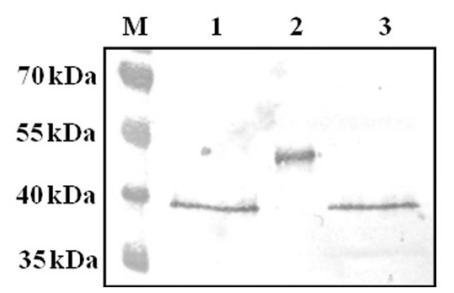

Figure 4. Expression of $\mathrm{HA} 1$ in plants.

(A) Schematic diagram of the HA1subunit cloned into 3' provector module. (B) Detection of HA1APO and HA1ER in Nicotiana benthamiana leaves by Western blot using monoclonal anti-polyHistidine antibody (mouse lgG, Sigma). Protein extracts from plant samples collected on selected days as indicated (6-8 dpi); K(-) extract from plants infiltrated with buffer. (C) De-glycosylation of HAER. Purified HAER were digested with Endo $\mathrm{H}$ (New England Biolabs) and PNGase (New England Biolabs), separated on SDS PAGE and detected by Western blot analysis with monoclonal anti-polyHistidine antibody. Lines 1-3, Endo $\mathrm{H}-$, undigested, PNGase-digested HAER, respectively. M- pre-stained molecular marker (Thermo Scientific).

from H5N1 (A/swan/Poland/305-135V08/2006) virus. The recombinant protein was targeted either to apoplast (HA1APO) or to ER (HA1ER) and contained a His-tags. In both cases higher than expected molecular weight of the recombinant protein was observed, due to $\mathrm{N}$-glycosylation. The proteins were partially purified by affinity chromatography using Ni NTA agarose under native or denaturing conditions. The highest amount of purified HA1 was obtained for HA1ER purified under native condition, $(3.33 \mathrm{mg} / \mathrm{kg} \mathrm{FW})$, while the amount of HA1APO was about four times lower $(0.83 \mathrm{mg} / \mathrm{kg}$ FW) (Fig. 4). Reactivity of purified HA with monoclonal anti-HA (H5N1) antibody purified from hybridoma cells (Mab 6-9-1, Institute of Biotechnology and Antibiotics, Warsaw, Poland) was tested by ELISA using Ni-NTA HisSorb Strips which allow antigen immobilization on the well surface by His-tag that, in turn, leads to a uniform orientation of the His-tagged antigens. The ELISA results confirmed specific interaction of plant-produced HA1 with anti-HA H5N1 antibody. We would like to emphasize that the applied method allows fast and efficient production of HA and/or its fragments, which can be applied for characterization and detection of anti-HA antibodies, for example to replace the Escherichia coli-produced proteins in such devices as electrochemical immunosensors (Jarocka et al., 2014).

Comparable results were reported by another group (Spitsin et al., 2009). In either stable or transient systems they obtained various versions of HA from H5N1 (A/Vietnam/1203/2004), including the full-length HA (1-549 aa), a shorter version containing only major an- tigenic domains (1-330 aa) either alone or in a fusion with Fc (human or mouse), a version truncated at C-terminal part (1-277 aa) and the same version additionally lacking the N-terminal region (68-277aa). The production level of HA1-Fc in MagnICON ${ }^{\circledR}$ system was about $1 \mathrm{mg} / \mathrm{kg}$ FW. However, after purification by protein-A (affinity) chromatography the yield decreased by $50 \%$. Interestingly, although significant humoral immune responses were observed in mice immunized with various HA1 variants, none of them induced virus-neutralizing ( $\mathrm{VN}$ ) antibodies.

\section{Alfalfa plastocyjanin gene expression cassette}

This system is based on an expression cassette containing upstream and downstream regulatory elements of the alfalfa plastocyanin gene, namely its promoter and terminator, and its 5'- and 3'-UTRs (D'Aoust et al., 2008). The strategy described below led to generation of recombinant VLPs in this system. The sequences encoding either fulllength $\mathrm{H} 5 \mathrm{HA}$ from the pandemic $\mathrm{H} 5 \mathrm{~N} 1$ (A/Indonesia/5/05) virus or the full-length H1 HA from the seasonal H1N1 (A/ New Calzedonia/20/99) were cloned into the plastocyanin-based expression cassette (D'Aoust et al., 2008). In H5-based construct the native signal peptide was used, while in $\mathrm{H} 1$-based one it was replaced by the signal peptide from the disulphide isomerase from alfalfa. Additionally, the plasmid carrying sequence encoding matrix protein M1 from H1N1 (A/Puerto Rico/8/34) was co-infiltrated along with $\mathrm{HA}$ constructs in order to facilitate the formation of VLPs. Interestingly, transmission electron microscopy confirmed formation of VLPs in extracts from plants agroinfiltrated with HA constructs alone, while co-expression of $\mathrm{HA}$ and M1 led to undesirable results with a substantial decrease of HA accumulation. The VLPs were located in apoplastic indentations of the plasma membrane. Immunization of mice with two doses of $0.1 \mu \mathrm{g}$ of purified H5-VLPs along with Alhydrogel ${ }^{\circledR}$ (adjuvant) induced a strong immune response against a homologous virus, whereas two higher doses $(0.5 \mu \mathrm{g})$ protected against a lethal challenge with the heterologous strain of H5N1 (A/Vietnam/1194/04).

\section{The $\mathrm{PEAQ}$ vector series}

This system is based on full-length or deleted versions of a Cowpea mosaic virus (CPMV) RNA-2, permitting efficient and rapid production of protein without viral replication (Sainsbury et al., 2009). A series of small binary vectors, named $\mathrm{pEAQ}$, which may be used for the production of a wide variety of protein in both, transient and stable expression systems, were engineered. These vectors contain $35 \mathrm{~S}$ CaMV promoter, nopaline synthase (nos) terminator, P19 sequence encoding suppressor of silencing, and 5'- and 3'-UTRs from CPMV RNA-2. The gene of interest is inserted between the UTRs. A new generation vector, pCPMV-HT, provides extremely high translational efficiency and, consequently, a high production level of the recombinant protein (Peyret \& Lomonossoff, 2013). 


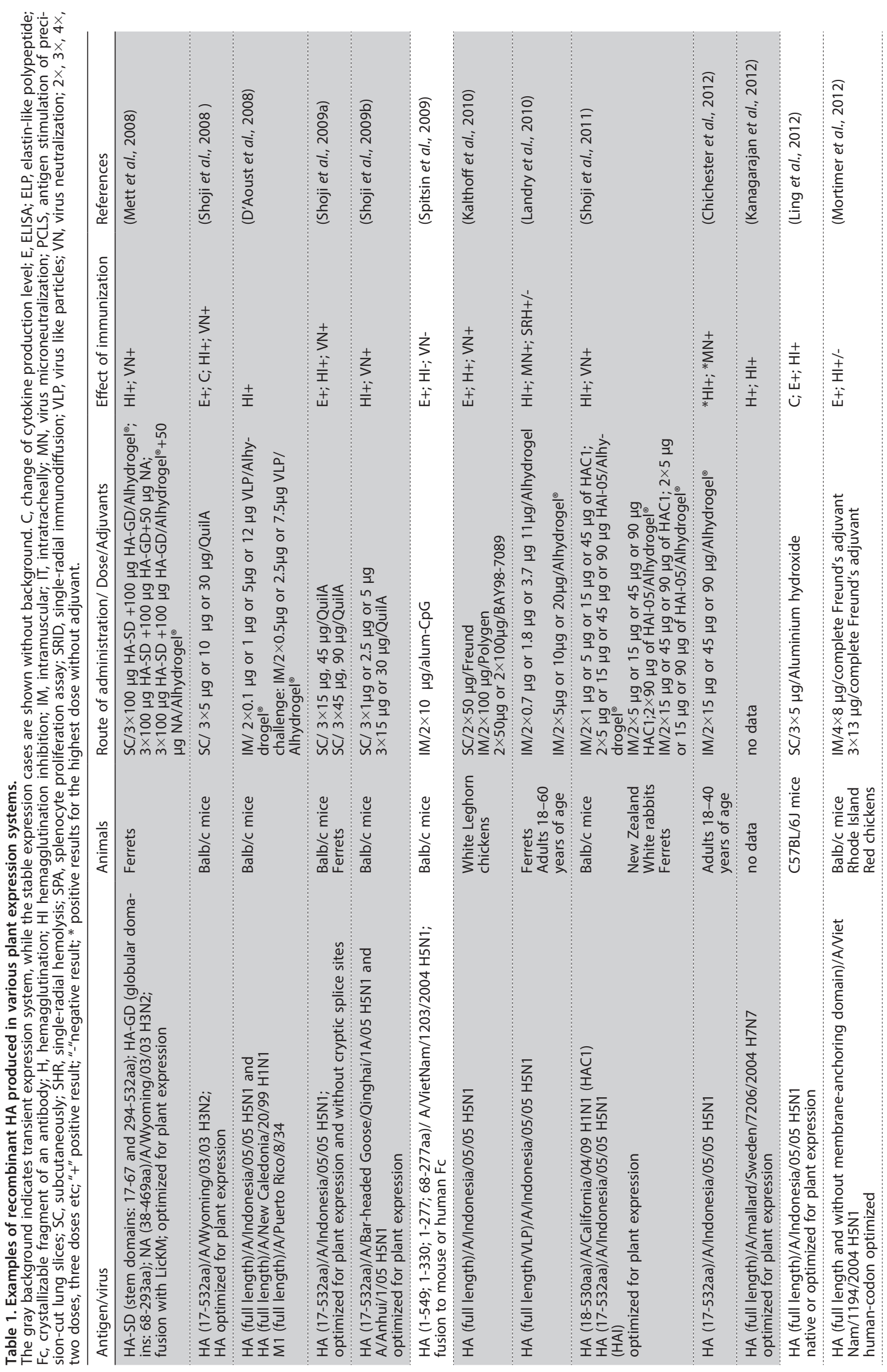




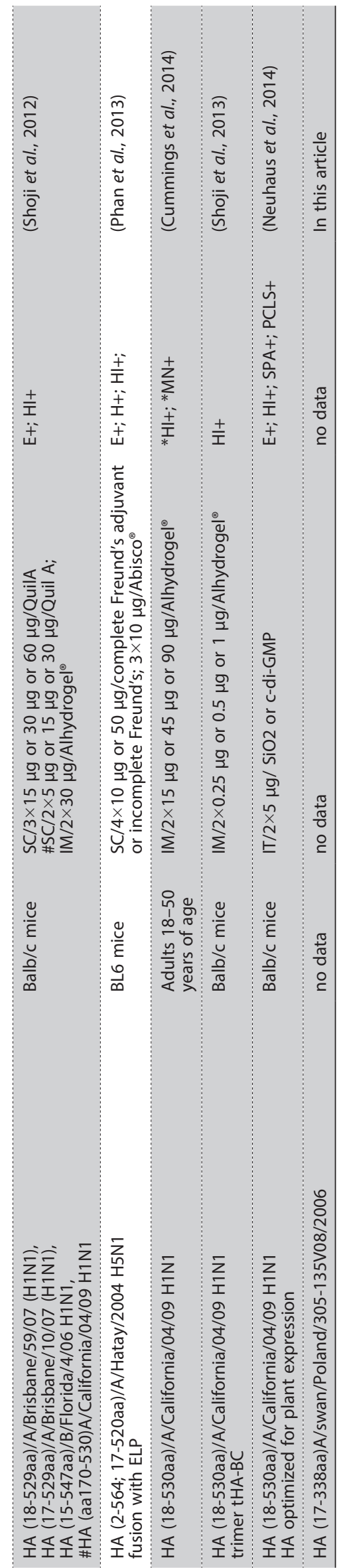

This system was chosen for industrial-scale (commercial) production of H5 VLPs. The co-expression of suppressor silencing or other helper proteins (HSP40 and HSP70) also significantly increased, especially in the case of H3 from H3N2 (A/Brisbane/10/07 H3N2), the VLPs accumulation (D'Aoust et al., 2010).

This system was also used for production of a biologically active full-length HA from H7N7 low pathogenicity avian influenza (LPAI) virus isolated from wild Swedish mallard ducks (Kanagarajan et al., 2012). Recombinant H7 HA was targeted to ER and the yield of production was about $200 \mathrm{mg}$ of purified protein per $\mathrm{kg}$ of FW.

\section{STABLE EXPRESSION}

Stable plant expression systems are used less often for the production of recombinant HA (Table 1). To achieve the high expression levels, the expression cassettes with strong promoters and regulatory elements enhancing transcription and translation are used. For example, a series of pTRA binary vectors containing 35S CaMV promoter with duplicated transcriptional enhancer, chalcone synthase 5' untranslated region (CHS UTR) and 35S CaMV polyadenylation signal (pA35S) have been created. Additionally, in PTRA vectors two copies of the scaffold attachment region (SAR) from tobacco Rb7 (root specific gene) flank the expression cassette. Another example of a series of plant expression vectors is the Impact vector $^{\text {TM }}$ system created by Plant Research International of Wageningen University and Research Center. These plasmids contain a very strong, light regulated Rubisco promoter. Both series of vectors enable targeting of the recombinant proteins into different subcellular compartments.

The pTRA vectors were used to set up the plantbased platform for influenza vaccine production in South Africa (Mortimer et al., 2012). Two recombinant variants, namely full-length and truncated form of HA from H5N1 (A/Vietnam/1194/2004) were successfully produced in different cellular compartments (cytoplasm, chloroplast, ER, and apoplastic spaces). The highest production level was observed for full-length HA targeted to apoplast (640-1440 mg/kg FW), and for truncated HA targeted to ER (160-880 mg/kg FW). Both forms of protein were purified and used in an immunization experiment, resulting in a serum from vaccinated chickens and mice containing specific anti-H5 HA antibody.

Fusion with elastin-like polypeptide (ELP) is supposed to increase the yield and improve the purification yield (Phan et al., 2013). Both monomeric and trimeric HA (ectodomain, 17-520 aa), with and without ELP polypeptide, were generated in transgenic $N$. tabacum plants and in transiently transformed $N$. benthamiana. To generate the trimeric form of HA, the trimeric motif from GCN4-pII was fused to the $\mathrm{C}$ terminus of HA ectodomain. ELPylation increased accumulation of the recombinant $\mathrm{HA}$ in plant leaves without influencing the functionality and the antigenicity of the HA trimers. Immunization of mice indicated that the trimeric form of $\mathrm{HA}$ induced a higher HA-specific immune response than the monomeric form, regardless of the presence or absence of ELP.

Polypeptide variants of the HA from $\mathrm{H} 5 \mathrm{~N} 1$ were produced in MagnICON ${ }^{\circledR}$ system (see above) and in transgenic plants using ImpactVector systems (Spitsin et al., 2009). Interestingly, expression of chimeric protein HA1Fc in transgenic plants was 4-fold higher than in transient system and reached $4 \mathrm{mg} / \mathrm{kg} \mathrm{FW}$. In comparison 
with HA1 alone (without Fc) the overall yield of production and purification stage requiring only one step was improved, but fusion-related enhancement of the immunogenicity in vaccinated mice was not detected (Spitsin et al., 2009).

\section{INTERSPECIFIC NICOTIANA HYBRID}

The main disadvantage of Nicotiana sp. plants as bioreactors for production of recombinant pharmaceuticals is a high concentration of toxic pyridine alkaloids, which precludes direct application of the crude plant extract or even only partially purified proteins. To get around this problem Ling and coworkers (Ling et al., 2012) engineered interspecific Nicotiana hybrid by crossing two transgenic lines, Nicotiana tabacum LAFC-HA producing recombinant HA and Nicotiana glauca A622-RNAi carrying a construct to downregulate alkaloid biosynthesis genes. The hybrid plants expressed HA and did not synthesize alkaloids in leaves. Importantly, these plants can be easily propagated by vegetative cuttings and did not produce viable pollen, which eliminates potential problems with bio-contamination. The crude extract from hybrid plants was used for mice immunization. It appeared to be nontoxic and elicited a high HA-specific IgG antibody titer as confirmed by ELISA. Moreover, analysis of $\mathrm{TNF} \alpha$ (tumor necrosis factor $\alpha$ ) and IFN $\alpha$ induction after incubation of the peripheral mononuclear cells with either purified HA or the crude extracts from the hybrid or control plants indicated that the plant extracts induced a stronger cytokine production than the purified HA (Ling et al., 2012).

\section{PURIFICATION OF RECOMBINANT HA}

Over $90 \%$ of the total cost of recombinant subunit vaccine production is related to the downstream processing, namely the separation and purification steps (Conley et al., 2011). Plant-produced recombinant HA has been purified by various methods prior to vaccination. The first step after plant tissue homogenization was filtration through Miracloth. Next, if the His-tag was present (in most cases), the partial purification by immobilized metal affinity chromatography was performed (Spitsin et al., 2009; Shoji et al., 2008; Mortimer et al., 2012). Anion exchange chromatography (Shoji et al., 2009b; Shoji et al., 2009a; Mett et al., 2008; Shoji et al., 2012) and ultrafiltration, followed by diafiltration and filter sterilization with a $0.2 \mu \mathrm{m}$ membrane were also used (Shoji et al., 2011). Mortimer and coworkers (Mortimer et al., 2012), besides affinity purification on Ni-NTA resin, applied also diafiltration using hollow fiber cartridges with a $50 \mathrm{kDa}$ membrane cut off.

The process of selective extraction followed by twophase separation and gel filtration was used to purify full-length HA expressed in a MagnICON ${ }^{\circledR}$ system (Kalthoff et al., 2010) while H5-VLPs purification consisted of several steps starting with homogenization and centrifugation, followed by a clarification step based on chemical and physical treatments. The clarified extract was concentrated and stabilized, then the proteins were isolated through a series of chromatographic steps including ion exchange and affinity chromatography and concentrated by cross-flow filtration and sterilized by filtration (D'Aoust et al., 2010).

Recombinant HAs were also expressed as chimeric proteins to facilitate purification, as a fusion with Fc fragment allowing a single-step purification using protein
A (Spitsin et al., 2009) or as a fusion with elastin-like polypeptide (ELP) enabling extraction of the fusion protein using membrane-based inverse transition cycling (Phan et al., 2013). The second strategy not only improved protein purification but also reduced its cost. ELP are oligomeric repeats of the pentapeptide Val-Pro-Gly-Xaa-Gly (where Xaa is any amino acid except Pro) that undergo a reversible inverse temperature transition. ELP is monomeric and soluble at a temperature below the transition temperature $(\mathrm{Tt})$, while it aggregates and becomes insoluble at a temperature above Tt. This strategy was used to purify monomeric and trimeric HA (Phan et al., 2013).

\section{CLINICAL TRIALS OF PLANT-DERIVED SUBUNIT VACCINES AGAINST INFLUENZA VIRUS}

Recombinant proteins used in pharmaceutical applications require certain quality standards. The good manufacturing practice (GMP) grade is obligatory for clinical purposes. At least two large biomanufacturing facilities are capable of plant-derived HA production under GMP conditions, Fraunhofer USA Center for Molecular Biotechnology (FhCMB) from USA and Medicago Inc. from Canada.

A large scale production of $\mathrm{H} 1 \mathrm{HA}$ (HAC1) and $\mathrm{H} 5$ HA (HAI-05) from H1N1 (A/California/04/09) and H5N1 (A/Indonesia/05/05), respectively, has been reported by the FhCMB group (Shoji et al., 2011). Recombinant proteins were produced under GMP conditions in $N$. benthamiana using transient expression strategy based on the 'launch vector'. Physicochemical and immunochemical properties of the purified HAC1 (average yield $90 \mathrm{mg} / \mathrm{kg} \mathrm{FW}$ ) and HAI-05 (average yield $50 \mathrm{mg}$ / $\mathrm{kg}$ FW) were characterized and their immunogenicity and safety was evaluated in pre-clinical studies in mice, rabbits and ferrets (Shoji et al., 2011; Chichester et al., 2012). Subsequently, both subunit vaccines were demonstrated to be safe and immunogenic in healthy human volunteers during Phase I of clinical trials (Chichester et al., 2012; Cummings et al., 2014). In both cases vaccine was administered intramuscularly, twice at three week intervals, to healthy adults of 18-50 years old. Three doses $(15,45$ and $90 \mu \mathrm{g})$ with or without Alhydrogel ${ }^{\circledR}$ (adjuvant) were tested. The highest response, detected by HI and VN antibody titers, was observed in the group immunized with the highest dose $(90 \mu \mathrm{g})$ without adjuvant.

According to the data deposited on the Medicago Inc. website (http://www.medicago.com/), three technologies were developed by the company for production of vaccines in the plant systems: (i) the Profi$\mathrm{cia}^{\mathrm{TM}}$ technology based on transient protein expression in plant leaves; (ii) Virus-Like Particles (VLPs) and (iii) the VLPExpress ${ }^{\mathrm{TM}}$ platform that accelerates the discovery and development of new vaccines by rapid expression, purification and testing of candidate VLPs. Using these technologies, Medicago Inc. has successfully produced H5 HA-based virus-like-particles (VLPs) derived from H5N1 (A/Indonesia/5/05) virus in $N$. benthamiana (Landry et al., 2010). Immunogenicity of the HA VLP vaccine was confirmed by the HI test, single radial hemolysis and microneutralisation. In the pre-clinical trials the alum adjuvanted plant-made VLPs induced production of cross-reactive antibodies in ferrets. In the Phase I of clinical trials, healthy adults of 18-60 years old were vaccinated intramuscularly twice, 21 days apart, with three different doses (5, 10 and $20 \mu \mathrm{g})$ of alum-adjuvanted H5 VLP vaccine or with a placebo. The vaccine was well tolerated at all tested doses. Additionally, the 
immunogenicity of plant-specific glycans was studied in humans during these trials and there was no induction of antibodies directed against plant $\mathrm{N}$-glycans (Landry et al., 2010). Medicago Inc. also successfully expressed a new VLP antigen (H1 VLP) based on H1 HA from H1N1 influenza virus A using the Company's VLP vaccine and Proficia manufacturing technologies. Recombinant antigen was expressed within 14 days after receiving the DNA sequence and induced a positive immune response in $100 \%$ of the vaccinated animals. Even a single dose of $5 \mu \mathrm{g}$ of HA VLP vaccine was inducing a response against the H1N1 (A/California/04/09) virus. In June 2011, Medicago completed a Phase I clinical trial with the candidate VLP vaccine ("H1N1 vaccine"). All doses tested were safe, well tolerated and induced a solid immune response that met the criteria of The Committee for Medicinal Products for Human Use (CHMP). Therefore, Medicago Inc. intends to proceed with a U.S. Phase IIa trial for its seasonal trivalent vaccine with antigens from the recommended $\mathrm{H} 1 \mathrm{~N} 1, \mathrm{H} 3 \mathrm{~N} 2$ and $\mathrm{B}$ influenza strains. The company also completed a Phase II clinical trial with the H5 VLP candidate against pandemic flu (H5N1). The candidate vaccine was applied intramuscularly or intradermally with Glucopyranosyl lipid A and was well tolerated (http://www.medicago.com).

\section{CONCLUSIONS AND PERSPECTIVES}

Data published on the WHO website (http://who.int/ immunization/diseases/influenza/clinical_evaluation_tables/en/.containing) concerning influenza candidate vaccines under clinical trials clearly indicate that most of the tested candidates for pandemic and potentially pandemic vaccines are either inactivated virions, inactivated split viruses or live attenuated viruses produced in embryonated eggs or in Vero cells. Only about $5 \%$ consist of recombinant vaccines produced in SP9 insect cells, chicken embryonic fibroblasts (CEF), E. coli and plants. It is not surprising, since the egg-based vaccine production is the oldest and the best-established technology, while the modern systems for production of the second generation vaccines need more time for recognition. However, considering limitations of the egg-based manufacturing system and the nature of influenza virus leading to rapid emergence of potentially pandemic strains, it is really important to develop alternative production systems. Positive results of the Phase I and Phase II clinical trials with plant-derived monomeric HA or VLPs confirmed that plant-based production platforms are reliable. Particularly noteworthy are transient expression systems, especially in emergency situations, such as pandemics. These systems are well established, less controversial (no genetically modified plants involved) and their most important advantage is speed. Medicago Corp. announced that it is able to produce 10 million doses of H1N1 VLP influenza vaccine candidate in one month. In current traditional manufacturing technologies that relay on strain adaptation, this period lasts 4-6 months. However, we should remember that the traditional vaccines, which are composed of the whole inactivated virus are more immunogenic and only one dose of vaccine is routinely used. Thus, reduction of the number of administrated doses and development of needle-free, easy to apply routs of administration should be the most important challenges for these novel technologies. Indeed, combination of the short production time with high effectiveness, low dose costs and comfort of vaccination would give a competitive advantage to the plant-based vaccines.

\section{Acknowledgements}

We are grateful to Dr. Victor Klimyuk (Icon Genetics $\mathrm{GmbH}$ ) for the MagnICON ${ }^{\circledR}$ vectors and instructions. We thank Anna Porębska, Violetta Cecuda-Adamczewska, Grażyna Płucieniczak and Violetta Sączyńska from the Institute of Biotechnology and Antibiotics (Warsaw, Poland) for supplying the hybridoma culture producing Mab 6-9-1.

\section{Acknowledgements of financial support}

This work was supported by the Ministry of Science and Higher Education, Poland, grant N302 061934 and in part by EU Innovative Economy Program, Grant No. WND-POIG.01.01.02-00-007/08.

\section{REFERENCES}

Chichester JA, Jones RM, Green BJ, Stow M, Miao F, Moonsammy G, Streatfield SI, Yusibov V (2012) Safety and immunogenicity of a plant-produced recombinant hemagglutinin-based influenza vaccine (HAI-05) derived from A/Indonesia/05/2005 (H5N1) influenza virus: a phase 1 randomized, double-blind, placebo-controlled, doseescalation study in healthy adults. Viruses 4: 3227-3244.

Conley AJ, Joensuu JJ, Richman A, Menassa R (2011) Protein bodyinducing fusions for high-level production and purification of recombinant proteins in plants. Plant Biotechnol J 9: 419-433.

Cummings JF, Guerrero ML, Moon JE, Waterman P, Nielsen RK, Jefferson S, Gross FL, Hancock K, Katz JM, Yusibov V, Group FUCfMBS (2014) Safety and immunogenicity of a plant-produced recombinant monomer hemagglutinin-based influenza vaccine derived from influenza A (H1N1)pdm09 virus: A Phase 1 dose-escalation study in healthy adults. Vaccine 32: 2251-2259.

D'Aoust MA, Couture MM, Charland N, Trépanier S, Landry N, Ors F, Vézina LP (2010) The production of hemagglutinin-based viruslike particles in plants: a rapid, efficient and safe response to pandemic influenza. Plant Biotechnol J 8: 607-619.

D’Aoust MA, Lavoie PO, Couture MM, Trépanier S, Guay JM, Dargis M, Mongrand S, Landry N, Ward BJ, Vézina LP (2008) Influenza virus-like particles produced by transient expression in Nicotiana benthamiana induce a protective immune response against a lethal viral challenge in mice. Plant Biotechnol J 6: 930-940.

Gleba Y, Klimyuk V, Marillonnet S (2005) Magnifection - a new platform for expressing recombinant vaccines in plants. Vaccine 23: 2042-2048.

Goldenkova IV, Musiǐchuk KA, Piruzian ES (2002) A thermostable Clostridium thermocellum lichenase-based reporter system for studying the gene expression regulation in prokaryotic and eukaryotic cells. Mol Biol (Mosk) 36: 868-876.

Jarocka U, Sawicka R, Gora-Sochacka A, Sirko A, Zagorski-Ostoja W, Radecki J, Radecka H (2014) Electrochemical immunosensor for detection of antibodies against influenza A virus $\mathrm{H} 5 \mathrm{~N} 1$ in hen serum. Biosensors \& Bioelectronics 55: 301-306.

Kalthoff D, Giritch A, Geisler K, Bettmann U, Klimyuk V, Hehnen HR, Gleba Y, Beer M (2010) Immunization with plant-expressed hemagglutinin protects chickens from lethal highly pathogenic avian influenza virus H5N1 challenge infection. J Virol 84: 12002-12010.

Kanagarajan S, Tolf C, Lundgren A, Waldenström J, Brodelius PE (2012) Transient expression of hemagglutinin antigen from low pathogenic avian influenza A (H7N7) in Nicotiana benthamiana. PLoS One 7: e33010.

Landry N, Ward BJ, Trépanier S, Montomoli E, Dargis M, Lapini G, Vézina LP (2010) Preclinical and clinical development of plantmade virus-like particle vaccine against avian H5N1 influenza. PLoS One 5: e15559.

Ling HY, Edwards AM, Gantier MP, Deboer KD, Neale AD, Hamill JD, Walmsley AM (2012) An interspecific Nicotiana hybrid as a useful and cost-effective platform for production of animal vaccines. PLoS One 7: e35688.

Marillonnet S, Giritch A, Gils M, Kandzia R, Klimyuk V, Gleba Y (2004) In planta engineering of viral RNA replicons: efficient assembly by recombination of DNA modules delivered by Agrobacterium. Proc Natl Acad Sci U S A 101: 6852-6857.

Mett V, Musiychuk K, Bi H, Farrance CE, Horsey A, Ugulava N, Shoji Y, de la Rosa P, Palmer GA, Rabindran S, Streatfield SJ, Boyers A, Russell M, Mann A, Lambkin R, Oxford JS, Schild GC, Yusibov V (2008) A plant-produced influenza subunit vaccine protects ferrets against virus challenge. Influenza Other Respir Viruses 2: 33-40.

Mortimer E, Maclean JM, Mbewana S, Buys A, Williamson AL, Hitzeroth II, Rybicki EP (2012) Setting up a platform for plant-based 
influenza virus vaccine production in South Africa. BMC Biotechnol 12: 14.

Musiychuk K, Stephenson N, Bi H, Farrance CE, Orozovic G, Brodelius M, Brodelius P, Horsey A, Ugulava N, Shamloul AM, Mett V, Rabindran S, Streatfield SJ, Yusibov V (2007) A launch vector for the production of vaccine antigens in plants. Influenza Other Respir Viruses 1: 19-25.

Neuhaus V, Chichester JA, Ebensen T, Schwarz K, Hartman CE, Shoji Y, Guzmán CA, Yusibov V, Sewald K, Braun A (2014) A new adjuvanted nanoparticle-based H1N1 influenza vaccine induced antigen-specific local mucosal and systemic immune responses after administration into the lung. Vacine 32: 3216-3222.

Obembe OO, Popoola JO, Leelavathi S, Reddy SV (2011) Advances in plant molecular farming. Biotechnol Adv 29: 210-222.

Peyret H, Lomonossoff GP (2013) The pEAQ vector series: the easy and quick way to produce recombinant proteins in plants. Plant Mol Biol 83: 51-58.

Phan HT, Pohl J, Floss DM, Rabenstein F, Veits J, Le BT, Chu HH, Hause G, Mettenleiter T, Conrad U (2013) ELPylated haemagglutinins produced in tobacco plants induce potentially neutralizing antibodies against H5N1 viruses in mice. Plant Biotechnol J 11: 582-593.

Sainsbury F, Thuenemann EC, Lomonossoff GP (2009) pEAQ: versatile expression vectors for easy and quick transient expression of heterologous proteins in plants. Plant Biotechnol J 7: 682-693.

Shoji Y, Bi H, Musiychuk K, Rhee A, Horsey A, Roy G, Green B, Shamloul M, Farrance CE, Taggart B, Mytle N, Ugulava N, Rabindran S, Mett V, Chichester JA, Yusibov V (2009a) Plant-derived hemagglutinin protects ferrets against challenge infection with the $\mathrm{A} /$ Indonesia/05/05 strain of avian influenza. Vacine 27: 10871092.

Shoji Y, Chichester JA, Bi H, Musiychuk K, de la Rosa P, Goldschmidt L, Horsey A, Ugulava N, Palmer GA, Mett V, Yusibov V
(2008) Plant-expressed HA as a seasonal influenza vaccine candidate. Vaccine 26: 2930-2934.

Shoji Y, Chichester JA, Jones M, Manceva SD, Damon E, Mett V, Musiychuk K, Bi H, Farrance C, Shamloul M, Kushnir N, Sharma S, Yusibov V (2011) Plant-based rapid production of recombinant subunit hemagglutinin vaccines targeting $\mathrm{H} 1 \mathrm{~N} 1$ and $\mathrm{H} 5 \mathrm{~N} 1$ influenza. Hum Vaccin 7 (Suppl): 41-50.

Shoji Y, Farrance CE, Bautista J, Bi H, Musiychuk K, Horsey A, Park H, Jaje J, Green BJ, Shamloul M, Sharma S, Chichester JA, Mett V, Yusibov V (2012) A plant-based system for rapid production of influenza vaccine antigens. Influenza Other Respir Viruses 6: 204-210.

Shoji Y, Farrance CE, Bi H, Shamloul M, Green B, Manceva S, Rhee A, Ugulava N, Roy G, Musiychuk K, Chichester JA, Mett V, Yusibov V (2009b) Immunogenicity of hemagglutinin from A/Bar-headed Goose/Qinghai/1A/05 and A/Anhui/1/05 strains of H5N1 influenza viruses produced in Nicotiana benthamiana plants. Vaccine 27: 3467-3470.

Shoji Y, Jones RM, Mett V, Chichester JA, Musiychuk K, Sun X, Tumpey TM, Green BJ, Shamloul M, Norikane J, Bi H, Hartman CE, Bottone C, Stewart M, Streatfield SJ, Yusibov V (2013) A plant-produced $\mathrm{H} 1 \mathrm{~N} 1$ trimeric hemagglutinin protects mice from a lethal influenza virus challenge. Hum V accin Immunother 9: 553-560.

Spitsin S, Andrianov V, Pogrebnyak N, Smirnov Y, Borisjuk N, Portocarrero C, Veguilla V, Koprowski H, Golovkin M (2009) Immunological assessment of plant-derived avian flu $\mathrm{H} 5 / \mathrm{HA} 1$ variants. Vaccine 27: 1289-1292.

Stevens J, Blixt O, Tumpey TM, Taubenberger JK, Paulson JC, Wilson IA (2006) Structure and receptor specificity of the hemagglutinin from an H5N1 influenza virus. Science 312: 404-410. 